\title{
State Estimation of the Vinyl Acetate Reactor Using Unscented Kalman Filters (UKF)
}

\author{
N. TUDOROIU and K. KHORASANI* \\ *Department of Electrical and Computer Engineering, Concordia University, Montreal, Quebec H3G \\ 1M8 Canada, E-mails: tnicolae@excite.com, kash@ece..concordia.ca
}

\begin{abstract}
The main objective of our research is to develop several unscented transform techniques (UTT) to estimate the state of the nonlinear processes such as an improvement of an Extended Kalman Filter (EKF) approach. The Extended Kalman Filter (EKF) has become a standard nonlinear estimation technique in control systems and parameter estimation for nonlinear system identification. The Unscented Kalman Filter (UKF) developed in this paper is a superior alternative to the Exdended Kalman Filter for the most of estimation and control applications. We will try to figure out in this paper that the UKF algorithm performs slightly superior compared to EKF algorithm based on the state estimation of the nonlinear vinyl acetate reactor developed well in [1]. These results are encouraging for us and we want to explore the possibility of extension of its applicability to the other possible applications such as state and parameter estimation, neural network identification, and Fault Detection, Diagnosis and Isolation (FDDI) of the nonlinear control systems [5] - [6].
\end{abstract}

\section{INTRODUCTION}

In the last thirty years the Extended Kalman Filter (EKF) has become a standard technique for state and parameter estimation of nonlinear systems by simply linearizing the nonlinear model of the process:

$$
\begin{aligned}
& x(k+1)=f(x(k), u(k), w(k)) \\
& y(k)=g(x(k), u(k), v(k))
\end{aligned}
$$

such that the traditional linear Kalman Filter (KF) approach can be applied. However, even if the EKF still remains a widely used filtering strategy, the experience accumulated with it application during this period reveals some fleas such as its difficulties in implementation, tuning, and its reliability for systems which are almost linear on the time scale of the update interval. To eliminate such inconvenience we try in our research to find another alternative methods that may improve the performance of EKF algorithm. In our paper we develop the unscented transform technique such as the Unscented Kalman Filter (UKF) to show the superiority of this algorithm compared to EKF algorithm.

\section{The EKF Algorithm Review}

In this section we try to make a short review of the EKF algorithm. Given the noisy observation $y_{k}$, a recursive state estimation for $x_{k}$ is given by

$$
\hat{x}_{k}=\left(\text { prediction of } x_{k}\right)+K_{k}\left[y_{k}-\left(\text { prediction of } y_{k}\right)\right]
$$

This recursive equation provides the optimal minimum mean-squared error (MMSE) estimate for $x_{k}$ based on the assumption that the prior estimate $X_{k-1}$ and current observation $y_{k}$ are Gaussian random variables. Also the process and measurements noises $w(k), v(k)$ are assumed independent, zero mean and of covariance matrices $Q_{w}$ and $R_{v}$ respectively. At this stage the linearity assumption of the model. is not demanded. The optimal terms in this recursive equation are given by [2]:

$$
\begin{aligned}
& \hat{x}_{k}^{-}=E\left[f\left(\hat{x}_{k-1}, w_{k-1}\right)\right] \\
& K_{k}=P_{x_{k} y_{k}} P_{y_{k} y_{k}}^{-1} \\
& \hat{y}_{k}^{-}=E\left[g\left(\hat{x}_{k}^{-}, v_{k}\right)\right]
\end{aligned}
$$

where the optimal prediction of $x_{k}$ is designated by $\hat{x}_{k}$, and represents the expectation of a nonlinear function of the random variables $\hat{x}_{k-1}$ and $w_{k-1}$; similar interpretation for the optimal prediction $\hat{y}_{k}^{-}$. The optimal Kalman Filter gain $K_{k}$ is expressed as a function of posterior covariance matrices (with $\tilde{y}_{k}=y_{k}-\hat{y}_{k}^{-}$).

The Kalman Filter (EKF) calculates these quantities similar to the linear case, and can be viewed as an efficient method for analytically propagating a Gaussian random variable through linear system dynamics. For nonlinear models the EKF can be viewed as approximating the optimal terms as:

$$
\begin{aligned}
\hat{x}_{k}^{-} & \approx f\left(\hat{x}_{k-1}, \bar{w}\right) \\
K_{k} & \approx \hat{P}_{X_{k}, y_{k}} \hat{P}_{y_{k}, y_{k}}^{-1} \\
\hat{y}_{k}^{-} & \approx g\left(\hat{x}_{k}^{-}, \bar{v}\right)
\end{aligned}
$$


where predictions are approximated as simply the function of the prior mean value for estimates, and the covariance are determined by linearizing the dynamic state equations. In other words, in the EKF the state distribution is approximated by a Gaussian Random Variable which is then propagated analytically through the first-order linearization of the nonlinear system. Therefore EKF can be viewed as providing first-order approximations to the optimal terms. These approximations, however, can introduce large errors in the true posterior mean and covariance of the transformed Gaussian random variable, which may lead to suboptimal performance and sometimes divergence of the filter.

\section{UNSCENTED TRANSFORM TECHNIQUES (UTT)}

Compared to EKF the UKF addresses the approximation issues presented in the Section 2. The state distribution is similar represented by a Gaussian Random Variable, but now it is specified using a minimal set of carefully chosen sigma points. These sigma points completely capture the true mean and covariance of the Gaussian random variable, and when propagated through the true non-linear system, capture the posterior mean and covariance accurately until the 3rd order (Taylor series expansion) for any nonlinearity.

The Unscented Transformation (UT) [3]-[4], is a method for calculating the statistics of a random variable, which undergoes a nonlinear transformation. Consider propagating a random variable $\mathrm{x}$ (dimension $L$ ) through a nonlinear function, $y=g(x)$. Assume $x$ has mean $\bar{x}$ and covariance $P_{x}$. To calculate the statistics of $y$, we form a matrix $X$ of $2 L+1$ sigma vectors $X_{i}$ (with corresponding weights $W_{i}$ ), as follows

$$
\begin{aligned}
& X_{0}=\bar{x} \\
& X_{i}=\bar{x}+\left(\sqrt{(L+\lambda) \times P_{X}}\right)_{i}, i=1,2 \ldots, L \\
& X_{i}=\bar{x}-\left(\sqrt{(L+\lambda) \times P_{x}}\right)_{i-L}, i=L+1, \ldots, 2 L \\
& W_{0}^{m}=\frac{\lambda}{L+\lambda} \\
& W_{0}^{c}=\frac{\lambda}{L+\lambda}+\left(1-\alpha^{2}+\beta\right) \\
& W_{i}^{m}=W_{i}^{c}=\frac{1}{2 \times(L+\lambda)}, i=1,2 \ldots, 2 L
\end{aligned}
$$

where the parameter $\lambda$ is selected in deterministic manner

$$
\lambda=\alpha^{2} \times(L+\kappa)-L
$$

and represents a scaling parameter.

$\alpha$ is a constant which determines the spread of the sigma points around mean state value $\bar{x}$ and is usually set to a small positive value. $\kappa$ is a secondary scaling parameter which is usually set to $0, \quad \beta$ is used to incorporate prior knowledge of the distribution of $x$ (for Gaussian distribution, $\beta=2$ is optimal), and $\sqrt{\left.(L+\lambda) P_{X}\right)_{i}}$ is the $i$-th row of the matrix square root, calculated by using a stable numeric algorithm such as Choleski decomposition [3]-[4],[7]-[8].

The Unscented Transform determine the mean and covariance of system output $y$ by approximation, using a weighted sample mean and covariance of the posterior sigma points.

A simple example is shown in Figure 1 for a twodimensional system.

$$
\begin{aligned}
& Y_{i}=g\left(X_{i}\right) \\
& \bar{y} \approx \sum_{i=0}^{2 L} W_{i}^{m} Y_{i} \\
& P_{y} \approx \sum_{i=0}^{2 L} W_{i}^{C}\left[Y_{i}-\bar{y}\right]\left[Y_{i}-\bar{y}\right]^{T}
\end{aligned}
$$

The left plot shows the true mean and covariance propagation using Monte-Carlo sampling [3]-[4],[8]; the center plots show the results using a linearization approach as would be done in the Unscented Transform (UT); the right plots show the performance of the UT (note only 5 sigma points are required). The superior performance of the UT is obviously.

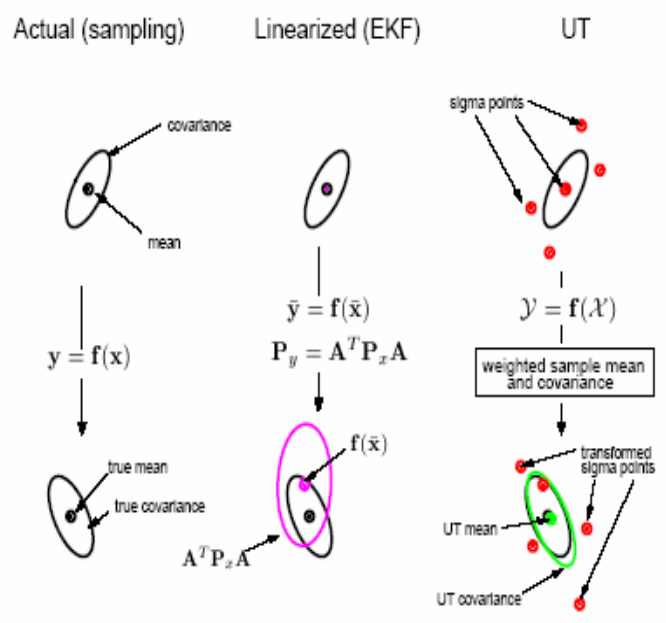

Fig. 1 Two-dimensional example of the Unscented Transform

\section{VINYL ACETATE REACTOR MODEL}

To establish the model of the Vinyl Acetate Reactor we start from the material balance and the energy balance of the reactor described by a chemical reaction of the type:

$$
A+B \rightarrow D
$$

where $A, B, D$ represent the acetylene, acetic acid reactants, and the vinyl acetate product respectively.

(i) Material Balance 


$$
\begin{aligned}
V \frac{d C_{A}}{d t} & =F\left(C_{A_{0}}-C_{A}\right)-K_{0} \exp \left(-\frac{E}{R T}\right) C_{A} C_{B} \\
V \frac{d C_{B}}{d t} & =F\left(C_{B_{0}}-C_{B}\right)-K_{0} \exp \left(-\frac{E}{R T}\right) C_{A} C_{B} \\
V \frac{d C_{D}}{d t} & =-F C_{D}+K_{0} \exp \left(-\frac{E}{R T}\right) C_{A} C_{B}
\end{aligned}
$$

(ii) Thermal Balance

$$
\rho V C \frac{d T}{d t}=V H K_{0} \exp \left(-\frac{E}{R T}\right) C_{A} C_{B}-h S\left(T-T_{a m}\right)+\rho F C\left(T_{0}-T\right)
$$

where $V$ is the reactor volume $\left[\mathrm{m}^{3}\right], C_{A}, C_{B}, C_{D}$ are the reactant and final product concentrations, $C_{A 0}, C_{B 0}$ are the initial concentrations of the reactants, $T, T_{0}$ are the inside and the output temperatures $\left[{ }^{0} K\right]$ of the vinyl acetate, $F$ is the supply mixed reactant flow $\left[\mathrm{m}^{3} / \mathrm{h}\right], T_{a m}$ is the average temperature of the thermal agent at the input and the output of the reactor $\left[{ }^{0} K\right], \rho, \rho_{a}$ are the densities of the supply mixed reactant and thermal agent $\left[\mathrm{kg} / \mathrm{m}^{3}\right], \mathrm{c}, \mathrm{c}_{\mathrm{a}}$ represent specific heat of the mixed reactant and thermal agent $\left[\mathrm{Kcal} / \mathrm{kg}{ }^{0} \mathrm{~K}\right]$, and $S$ represents the reactor surface $\left[\mathrm{m}^{2}\right], E=$ $5600[\mathrm{cal} / \mathrm{mol}], K_{0}=10^{5}\left[\mathrm{~m}^{3} / \mathrm{h}\right], \quad R=2\left[\mathrm{cal} / \mathrm{mol}{ }^{0} \mathrm{~K}\right]$, $H=20.800[\mathrm{Kcal} / \mathrm{Kg} \mathrm{mol}]$.

After some manipulations the vinyl acetate reactor model could be described by the following equations [1]

$$
\begin{aligned}
\frac{d x_{1}}{d t}= & -k_{1} \exp \left(-\frac{a}{x_{3}}\right) x_{1} x_{2}+k_{2} u_{1}\left(x_{10}-x_{1}\right) \\
\frac{d x_{2}}{d t}= & -k_{1} \exp \left(-\frac{a}{x_{3}}\right) x_{1} x_{2}+k_{2} u_{1}\left(1-x_{20}-x_{1}\right) \\
\frac{d x_{3}}{d t}= & -k_{3} \exp \left(-\frac{a}{x_{3}}\right) x_{1} x_{2}+k_{4} \frac{k_{5} u_{2}-k_{6} u_{2} x_{3}}{k_{6} u_{2}+k_{7}}+ \\
& +k_{2}\left(x_{30}-x_{3}\right) u_{1}
\end{aligned}
$$

where $x=\left[\begin{array}{lll}x_{1} & x_{2} & x_{3}\end{array}\right]^{T}$ is the vector state of the acetylene concentration $C_{A}$, acetic acid concentration $C_{B}$, and vinyl acetate temperature $T, u=\left[\begin{array}{ll}u_{1} & u_{2}\end{array}\right]^{T}$ represents the input vector. For the simulation the coefficients $a, k_{1}-k_{7}$ have been chosen with respect to the reactor geometry and have the following values:

$$
\begin{aligned}
& \mathrm{a}=2800, k_{1}=2.31, k_{2}=0.083, k_{3}=95.56, \\
& k_{4}=0.77, k_{5}=556870, k_{6}=1096.2, k_{7}=4480 .
\end{aligned}
$$

The set of the equations (11) is completed with the linear observation equation:

$$
y=1-x_{1}-x_{2}
$$

that represents the concentration of the vinyl acetate. To apply the UKF algorithm to this highly nonlinear model firstly we have to convert it in a discrete model, let assume the sampling time $\mathrm{T}_{\mathrm{s}}=1[\mathrm{~s}]$.

\section{THE UNSCENTED KALMAN FILTER ALGORITHM (UKF)}

Step 1: Initialization

$$
\begin{aligned}
& \hat{x}_{0}=E\left[x_{0}\right] \\
& P_{0}=E\left[\left(x_{0}-\hat{x}_{0}\right)\left(x_{0}-\hat{x}_{0}\right)^{T}\right]
\end{aligned}
$$

\section{Step 2: Computations}

For $k=1,2 \ldots, \mathrm{n}$ compute the sigma points matrix

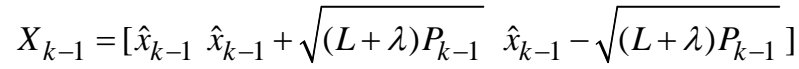

\section{Step 3: Time update}

$$
\begin{aligned}
& X_{k \mid k-1}=F\left[X_{k-1}, u(k)\right] \\
& \hat{x}_{k}^{-}=\sum_{i=0}^{2 L} W_{i}^{m} X_{i, k \mid k-1} \\
& P_{k}^{-}=\sum_{i=0}^{2 L} W_{i}^{c}\left[X_{i, k \mid k-1}-\hat{x}_{k}^{-}\right]\left[X_{i, k \mid k-1}-\hat{x}_{k}^{-}\right]^{T}+Q_{w} \\
& Y_{k \mid k-1}=H\left[X_{k \mid k-1}, u(k)\right] \\
& \hat{y}_{k}^{-}=\sum_{i=0}^{2 L} W_{i}^{m} Y_{i, k \mid k-1}
\end{aligned}
$$

Step 4: Measurement update

$$
\begin{aligned}
& P_{\tilde{y}_{k} \tilde{y}_{k}}=\sum_{i=0}^{2 L} W_{i}^{C}\left[Y_{i, k \mid k-1}-\hat{y}_{k}^{-}\right]\left[Y_{i, k \mid k-1}-\hat{y}_{k}^{-}\right]^{T} \\
& P_{x_{k} y_{k}}=\sum_{i=0}^{2 L} W_{i}^{C}\left[X_{i, k \mid k-1}-\hat{x}_{k}^{-}\right]\left[Y_{i, k \mid k-1}-\hat{y}_{k}^{-}\right]^{T}+R_{v} \\
& \kappa=P_{X_{k} y_{k}} P_{\tilde{y}_{k} \tilde{y}_{k}}^{-1} \\
& \hat{x}_{k}=\hat{x}_{k}^{-}+\kappa\left(y_{k}-\hat{y}_{k}^{-}\right) \\
& P_{k}=P_{k}^{-}-\kappa P_{\tilde{y}_{k} \tilde{y}_{k}} \kappa^{T}
\end{aligned}
$$

\section{SIMULATION RESULTS}

Based on the both EKF and UKF algorithms, we simulate the evolution of the true value and the estimated values. The results of these simulations are represented in Figures 1-3. In Figure 1 are represented the true value and the estimated values of the Acetylene concentration using the EKF and UKF algorithms. Similar in Figure 2 are represented the true value and estimated values of the Acetate Acid concentration, and in Figure 3 are represented the true value and estimated values of the temperature inside the reactor. Since for UKF algorithm, we don't need the linear model of the process, but only developing the sigma points from the initial assumptions. Hence UKF algorithm is more practical and accurate than 
EKF algorithm and the program implementation is more simple. In the Figures 1-2 we could observe the good tracking and convergence of the UKF algorithm compared to the EKF algorithm, and in Figure 3 we could see a good behavior of the both algorithms, for a bad guess of the initial condition for the reactor temperature.

\section{CONCLUSIONS}

In this paper, we develop the UKF algorithm to estimate the concentrations and the temperature of the Vinyl Acetate reactor and we compare these values with the results given by the EKF algorithm. The simulation results reveal a superior performance of the UKF algorithm compared to EKF algorithm. This approach is very interesting because doesn't need the linear model of the process that means time consuming and increasing the risk to reduce the accuracy in capturing the appropriate dynamics of the process.

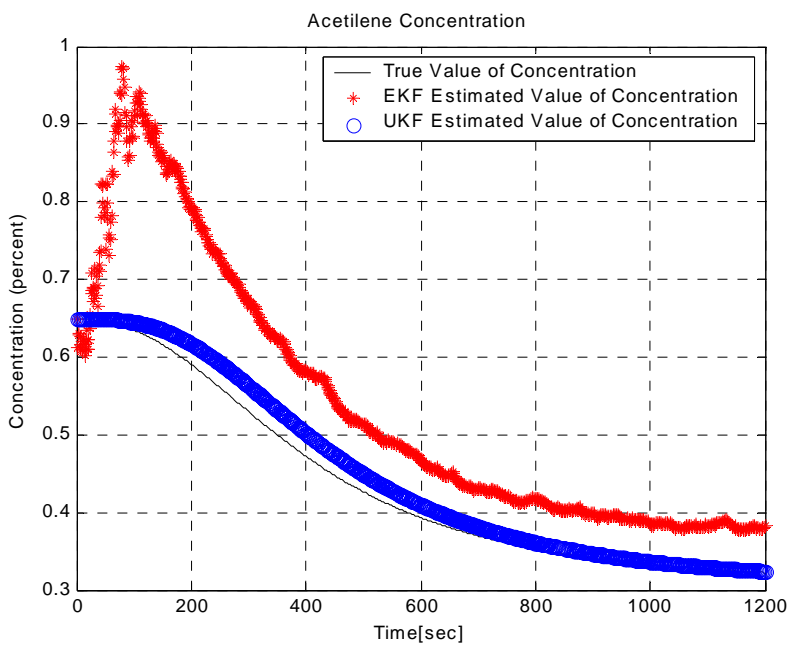

Fig. 1 The Acetylene Concentration

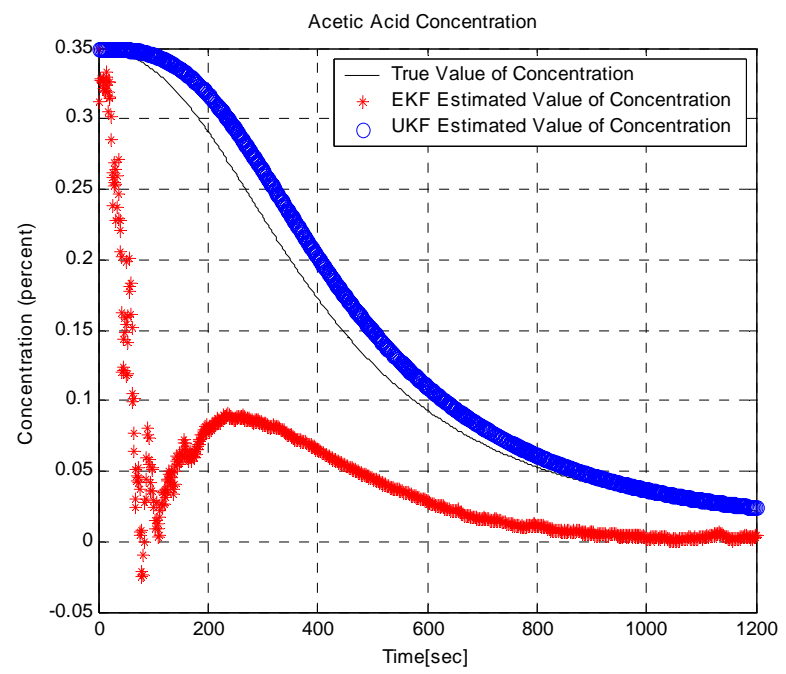

Fig. 2 The Acetate Acid Concentration

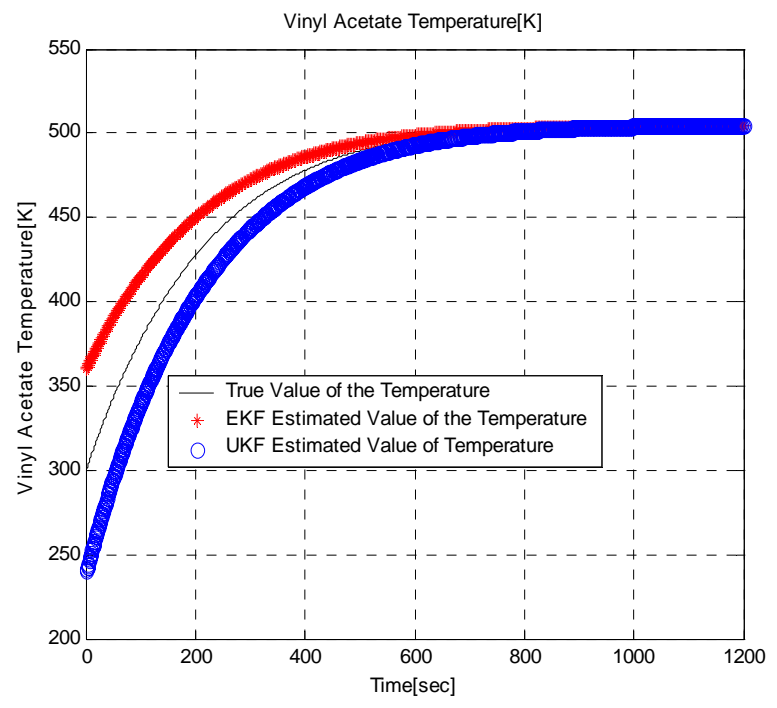

Fig. 3 The Vinyl Acetate Temperature

\section{REFERENCES}

[1] N.Tudoroiu, Chemical Nonlinear Processes Optimization, Ph. D Thesis, Romania, 1990.

[2] G.Plett, "Extended Kalman filtering for battery management systems of LiPB-based HEV battery packs. Part 2. Modeling and identification", J. Power Sources 134, 2004, pp. 262-276.

[3] S.Haykin, Adaptive filter theory - fourth edition, Prentice Hall, 2003.

[4] J.Joseph , LaViola Jr., "A Comparison of Unscented and Extended Kalman Filtering for Estimating Quaternion Motion”, Proceedings of the American Control Conference Denver, Colorado June 4-6, 2003.

[5] N.Tudoroiu, K.Khorasani,. "Fault Detection and Diagnosis for Reaction Wheels of Satellite's Attitude Control System Using a Bank of Kalman Filters", International Symposium on Signal, Circuits and Systems, ISSCS, Iasi, Romania, 2005, IEEE Proceedings, Vol. 1, pp. 199-202.

[6] N.Tudoroiu, K.Khorasani, "Fault Detection and Diagnosis for Satellite's Attitude Control System using an Interactive Multiple Model (IMM) Approach", The 2005 Conference on Control Applications, CCA05, Toronto, Canada, 28-31 August 2005, CD-ROM Proceedings.

[7] S.J.Julier, J.K.Uhlman, "A New Extension of the Kalman Filter to Nonlinear Systems”. Proceedings of AeroSense, The $11^{\text {th }}$ International Symposium on Aerospace/Defence Sensing, Simulation and Controls, 1997, pp.182-193.

[8] E.A.Wan, R.Merwe, "The Unscented Kalman Filter for Nonlinear Estimation”, Proceedings IEEE Symposium 2000 (AS-SPCC), Lake Louise, Alberta, Canada, Oct. 2000. 